\title{
Two-dimensional wave propagation without anomalous dispersion
}

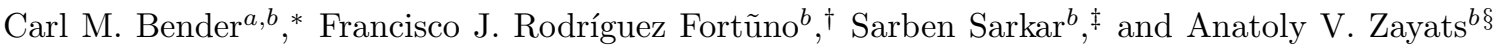 \\ ${ }^{a}$ Department of Physics, Washington University, St. Louis, MO 63130, USA \\ ${ }^{b}$ Department of Physics, King's College London, London WC2R 2LS, UK
}

(Dated: December 28, 2016)

\begin{abstract}
In two space dimensions and one time dimension a wave changes its shape even in the absence of a dispersive medium. However, this anomalous dispersive behavior in empty two-dimensional space does not occur if the wave dynamics is described by a linear homogeneous wave equation in two space dimensions and two time dimensions. Wave propagation in such a space can be realized in a three-dimensional anisotropic metamaterial in which one of the space dimensions has a negative permittivity and thus serves as an effective second time dimension. These results lead to a fundamental understanding and new approaches to ultrashort pulse shaping in nanostructures and metamaterials.
\end{abstract}

A light pulse traveling through a 3 -D medium ( $\mathrm{D}$ is the number of space dimensions) changes its shape and develops a tail as it propagates if different frequency components of the wave travel at different speeds. This pulse broadening, which is called dispersion, occurs in a medium with a frequency-dependent refractive index. Dispersion of light does not occur in 3 -D in a vacuum.

In contrast, in 2-D (three-dimensional space-time) a pulse changes its shape and forms a tail in a vacuum even though the wave speed $c$ is a frequency-independent constant. This dispersion in vacuum in 2-D cannot be explained in terms of a variable wave speed, and so this phenomenon is called anomalous dispersion [1, 2]. Anomalous dispersion in vacuum is a geometrical phenomenon associated with the dimension of space in which the waves propagate and it occurs for all kinds of waves, electromagnetic and acoustic. It occurs in two-dimensional but not in three-dimensional space.

The rumbling of thunder provides physical evidence of anomalous dispersion. A lightning bolt is a long (approximately translationally invariant) vertical line source. The sound waves that are produced propagate outward as two-dimensional cylindrical waves. Although the lightning bolt is instantaneous, an observer does not hear a "bang," but rather a rumbling that fades like $1 / t$.

In this Letter we show that by adding a second time-like coordinate to a three-dimensional space-time, anomalous dispersion in vacuum can be eliminated, leading to nondispersing pulse propagation. Such a nonMinkowskian space-time can be simulated by metamaterials with hyperbolic dispersion [3. These metamaterials have two space directions in which their effective permittivity is positive, and a third direction with a negative permittivity. Electromagnetic fields in a nondispersive and nonmagnetic anisotropic material with a uniaxial dielectric permittivity tensor $\overline{\bar{\varepsilon}}=\operatorname{diag}\left(\varepsilon^{x}, \varepsilon^{x}, \varepsilon^{z}\right)$ obey Maxwell's equations $\nabla \times \mathbf{E}=-\mu_{0} \mathbf{H}_{t}$ and $\nabla \times \mathbf{H}=\varepsilon_{0} \overline{\bar{\varepsilon}} \mathbf{E}_{t}$. These can be combined to give $\nabla \times \nabla \times \mathbf{E}=-\mu_{0} \varepsilon_{0} \overline{\bar{\varepsilon}} \mathbf{E}_{t t}$. This, together with Gauss' law $\nabla \cdot(\overline{\bar{\varepsilon}} \mathbf{E})=0$, gives the wave equation $\underline{3}$ ]

$$
u_{t t}=\frac{c^{2}}{\varepsilon^{x}}\left(u_{x x}+u_{y y}\right)+\frac{c^{2}}{\varepsilon^{z}} u_{z z},
$$

where the scalar field $u \equiv E^{z}$ represents the $z$ component of the electric field and the speed of light is given by $c^{2}=\left(\mu_{0} \varepsilon_{0}\right)^{-1}$. This equation becomes the usual homogeneous 3 -D wave equation when $\varepsilon^{x}=\varepsilon^{z}$, as is the case in vacuum. However, it can also represent the wave equation in two time and two space dimensions when $\varepsilon^{z}$ is negative and $\varepsilon^{x}$ is positive. Such materials exist and are known as hyperbolic materials; anisotropic composites with diagonal components of the effective permittivity tensor having opposite signs can be constructed as metal-dielectric multilayers or metal nanorod arrays 4 . Light propagates inside them as cones [5, 6] and their two-time character has been studied [3, 7, 8, raising the intriguing possibility of observing dispersionless propagation in 2-D. Space-times with two time dimensions also occur in M-theory [9].

The homogeneous linear wave equation

$$
u_{t t}=c^{2} \nabla^{2} u
$$

describes waves $u(\mathbf{x}, t)$ that travel with constant (frequency-independent) wave speed $c$ through a uniform medium. This wave equation describes how an initial pulse at $t=0$ given by the initial conditions

$$
u(\mathbf{x}, 0)=q(\mathbf{x}), \quad u_{t}(\mathbf{x}, 0)=p(\mathbf{x}),
$$

evolves into the wave $u(\mathbf{x}, t)$ at time $t$. This initial-value problem for the wave equation has an explicit quadrature solution in any space dimension.

Wave propagation in odd-dimensional space is fundamentally different from wave propagation in evendimensional space. When the space dimension $\mathrm{D}$ is odd and $\mathrm{D}>1$, waves obey Huygens' principle [1, 2]; that is, waves created by an instantaneous point source at $t=0$ (e.g., a light pulse) take the form of an expanding bubble. After the wavefront passes by, the medium instantly 
returns to quiescence. An observer sees blackness until the wave arrives, sees an instantaneous flash as the wave passes by, and immediately afterward sees blackness again [see Fig. 1(a)]. Such a wave propagates on the surface of the light cone. In contrast, in even-dimensional space an instantaneous point source gives rise to a wave that develops a tail. An observer sees blackness until the wave arrives and then sees a flash. However, the medium does not immediately return to quiescence; rather, the wave amplitude decays to 0 like $t^{-\alpha}$, where $\alpha>0$ depends on $\mathrm{D}$. When $\mathrm{D}=2$, the wave amplitude decays to 0 like $1 / t$. The tail of the wave propagates less rapidly than $c$ as a consequence of anomalous dispersion. Such a wave propagates on the surface and in the interior of the light cone [see Fig. 11(b)]. In a vacuum, if Huygens' principle applies, there is no anomalous dispersion [1, 2].

2-D wave propagation without anomalous dispersion. Anomalous dispersion occurs in vacuum in 2D. However, one might wonder whether it is possible to produce a medium whose dispersive properties exactly cancel the anomalous dispersion that occurs in 2-D wave propagation. Such a medium actually exists. The effect of anomalous dispersion can be cancelled if we modify the $2-\mathrm{D}$ wave equation by adding lower derivatives in time:

$$
u_{t t}-t^{-1} u_{t}+t^{-2} u=c^{2}\left(u_{x x}+u_{y y}\right) .
$$

The one-derivative term models gain, which speeds up the lagging tail of a 2-D wave. However, this term is too strong, so to reduce its effect we also introduce the time-dependent term $t^{-2} u$. For this artificial medium a flash bulb gives rise to a wave that does not disperse; the wave remains confined to the surface of the light cone and does not leak into the interior of the light cone [see Fig. 11(c)]. Below we demonstrate analytically this dispersionless propagation. Furthermore, we show that the wave equation (3) is equivalent to a constant-coefficient wave equation in two space and two time dimensions.

1-D homogeneous wave equation. The $1-\mathrm{D}$ wave equation is special because its solutions obey Huygens' principle when $p=0, q \neq 0$, and they exhibit anomalous dispersion when $q=0, p \neq 0$. The general solution $u(x, t)=f(x+c t)+g(x-c t)$ to the $1-\mathrm{D}$ wave equation is a superposition of two waves of unchanging shape traveling at constant speed $c$, one moving to the left and the other moving to the right. The exact solution satisfying the initial conditions (2) is given by D'Alembert's formula:

$$
u(x, t)=\frac{q(x+c t)+q(x-c t)}{2}+\frac{1}{2 c} \int_{x-c t}^{x+c t} d s p(s) .
$$

3-D homogeneous wave equation. The exact solution $u(\mathbf{x}, t)$ to the initial-value problem for the 3 -D wave equation $u_{t t}=c^{2}\left(u_{x x}+u_{y y}+u_{z z}\right)$ is obtained by using a construction invented by Kirchhoff. The quadrature solution is given compactly by Poisson's formula [1, 2]:

$$
u(x, y, z, t)=\frac{\partial}{\partial t}\left(t \omega_{c t}[q]\right)+t \omega_{c t}[p] .
$$

The spherical mean $\omega_{c t}[\phi]$ of the function $\phi(x, y, z)$ is an integral over the surface of a three-dimensional sphere of radius $c t$ centered at $(x, y, z)$ :

$$
\omega_{c t}[\phi] \equiv \int_{\alpha^{2}+\beta^{2}+\gamma^{2}=1} \frac{d \Omega}{4 \pi} \phi(x+c t \alpha, y+c t \beta, z+c t \gamma),
$$

where $d \Omega$ is an infinitesimal solid angle. Equations (5) and (6) are the complete solution to (1) and (2).

2-D homogeneous wave equation. The solution to the initial-value problem for the 2-D wave equation $u_{t t}=c^{2}\left(u_{x x}+u_{y y}\right)$ can be expressed in Poisson form (5), but now the 3-D spherical mean is a weighted average over the surface of a $2-\mathrm{D}$ disk centered at $(x, y)$ :

$$
\omega_{c t}[\phi] \equiv \frac{1}{2 \pi} \iint_{\alpha^{2}+\beta^{2} \leq 1} d \alpha d \beta \frac{\phi(x+c t \alpha, y+c t \beta)}{\sqrt{1-\alpha^{2}-\beta^{2}}} .
$$

We derive the integral in (7) from (6) by applying Hadamard's method of descent in which we project from $\mathrm{D}=3$ down to $\mathrm{D}=2$ by assuming that $\phi(x, y, z)$ is independent of $z$.

The method of descent may be used to project the 2-D solution (5) and (7) down to $\mathrm{D}=1$, allowing us to recover D'Alembert's solution (4) to the 1-D wave equation. To do so we choose $\phi(x, y)$ in (7) to be independent of $y$.

Verification of Huygens' Principle in 3-D. The 3 -D solution $u(x, y, z, t)$ in (5)-(6) depends on values of $q$ and $p$ only at points that are exactly a distance $c t$ from $(x, y, z)$. Points that are further from $(x, y, z)$ than $c t$ do not affect the solution $u(x, y, z, t)$ because wave disturbances from such points cannot travel faster than $c$. However, the fact that points that are closer to $(x, y, z)$ than $c t$ also do not affect the solution $u(x, y, z, t)$ is a surprise because there is ample time for waves emanating from these nearby points to reach the point $(x, y, z)$. It is this feature of 3 -D wave propagation that leads to Huygens' principle. As stated earlier, 1-D and 2-D wave propagation do not obey Huygens' principle.

Solution to the initial-value problem for a 3-D point disturbance. Consider a $3-\mathrm{D}$ medium that is initially quiescent and suppose that at $t=0$ there is a momentary light pulse at the origin. We represent such a disturbance by

$$
u(x, y, z, 0)=0, \quad u_{t}(x, y, z, 0)=\delta(x) \delta(y) \delta(z) .
$$

To see how this disturbance propagates in time, we substitute (8) into Poisson's formula (5) and evaluate the integral in (6). We obtain the spherical wave form

$$
u(x, y, z, t)=\frac{1}{4 \pi c r} \delta(r-c t),
$$

where $r=\sqrt{x^{2}+y^{2}+z^{2}}$. This 3-D wave resulting from the point disturbance (8) is precisely the expected expanding bubble. An observer at a distance $r$ from the 3 -D point disturbance waits a time $t=r / c$ and then 
detects a momentary flash followed by total quiescence. There is no remnant of this disturbance when $t>r / c$.

Anomalous dispersion in 1-D. Huygens' principle does not hold in 1-D because D'Alembert's solution (4) for $u(x, t)$ depends on $p(s)$ for $x-c t \leq s \leq x+c t$ and not just on $p(x+c t)$ and $p(x-c t)$. To see this, consider the evolution of a 1-D point disturbance at $x=0$ (a pulse), which we represent by the initial conditions $q(x)=u(x, 0)=0, p(x)=u_{t}(x, 0)=\delta(x)$. D'Alembert's formula (4) shows that these initial conditions spawn a wave in the form of a two-sided step function:

$$
u(x, t)=\frac{1}{2 c} \theta(c t-|x|) .
$$

An observer at $x$ must wait a time $t=|x| / c$ before the pulse arrives. After the wavefront passes, the medium does not return to its initially quiescent state; an upward displacement of $1 /(2 c)$ persists for all time. Thus, 1-D wave propagation violates Huygens' principle [1, 2].

There is a special class of initial conditions, $p(x)=$ $u_{t}(x, 0)=0$, that creates waves that $d o$ obey Huygens' principle. This is because waves arising solely from an initial displacement leave the medium quiescent after they have passed. For example, consider the initial conditions

$$
u_{t}(x, 0)=0, \quad u(x, 0)=\left\{\begin{array}{cc}
1-|x| & (|x|<1), \\
0 & (|x| \geq 1) .
\end{array}\right.
$$

These initial conditions correspond to an initially triangular transverse displacement and no initial transverse velocity. D'Alembert's formula shows that the initial pulse splits into a right-going and a left-going triangular pulse, each having half the initial amplitude. The two pulses travel to the right and to the left with speed $c$ and do not change their shape. The medium is quiescent until a pulse arrives, and after the pulse passes by, the medium returns to quiescence.

2-D waves and anomalous dispersion. To show that 2-D wave propagation does not obey Huygens' principle, we examine the effect of a 2-D point disturbance:

$$
u(x, y, 0)=0, \quad u_{t}(x, y, 0)=\delta(x) \delta(y) .
$$

This pulse disturbance is an idealization of the initial conditions created, for example, by a fish surfacing on a quiet pond. Substituting (10) into (7) and (5), we obtain

$$
u(x, y, t)=\frac{\theta\left(c^{2} t^{2}-x^{2}-y^{2}\right)}{2 \pi c \sqrt{c^{2} t^{2}-x^{2}-y^{2}}} .
$$

This formula describes a circular wave that propagates outward at speed $c$. An observer at $x^{2}+y^{2}=r^{2}$ must wait until time $t=r / c$ before the leading edge of the wave front arrives. The value of $u$ at the leading edge is infinite, but after the wavefront passes, the trailing wave decays like $1 / t$ for large $t$.

This explains why thunder rumbles, as mentioned previously. The surface of the earth is locally flat and twodimensional. The sound of thunder propagates across the surface in the same way that the wave in (11) propagates from a pointlike disturbance. A less idealized way to understand the rumbling of thunder is to view it as a 3-D wave in which the observer on the ground hears waves of the form (8) that emanate from successively higher and more distant points on the lightning bolt as $t$ increases. This explains the continued and tapering rumbling. Also, since the height of the lightning bolt is finite, after some time the rumbling abruptly stops.

Large-time asymptotic behavior of 2-D waves. The $1 / t$ decay in $2-\mathrm{D}$ space of a trailing wave, as in (11), is a general property of any wave created by a localized disturbance. To show this, substitute $a=c t \alpha$ and $b=$ ct $\beta$ into (7). Assuming that $\phi$ has compact support, then

$$
t \omega_{c t}[\phi] \sim \frac{1}{2 \pi c^{2} t} \iint_{R} d a d b \phi(a, b) \quad(t \rightarrow \infty) .
$$

If the integral in 12 exists and is nonzero, then $t \omega_{c t}[\phi] \propto$ $1 / t$ as $t \rightarrow \infty$.

Solution to the modified wave equation (3). This wave equation is singular at $t=0$, so we do not try to solve (3) for the general initial conditions (2). However, we impose the special initial conditions

$$
u(x, y, 0)=q(x, y)=0, \quad u_{t}(x, y, 0)=p(x, y)
$$

by following the Kirchhoff construction procedure. We rewrite (3) in spherical coordinates and seek radially symmetric solutions $u(x, t)$ to

$$
u_{t t}-t^{-1} u_{t}+t^{-2} u=c^{2}\left(u_{r r}+r^{-1} u_{r}\right) .
$$

We verify by direct differentiation (care is required) that

$$
u(r, t)=\frac{1}{2 \pi c} \delta(r-c t)
$$

exactly solves 13). By superposing solutions of this form, we construct a large class of solutions to (3) containing one arbitrary function of two arguments:

$$
u(x, y, t)=t \omega_{c t}[p],
$$

where

$$
\omega_{c t}[\phi] \equiv \frac{1}{2 \pi} \int_{0}^{2 \pi} d \theta \phi(x+c t \cos \theta, y+c t \sin \theta) .
$$

Equation $(15)$ is the exact solution to (3) for the initial conditions 2 with $q(x, y)=u(x, y, 0)=0$. Of course, (15) is not the general solution to (3) because the general solution contains two arbitrary functions of two arguments each. We cannot express the general solution to (3) in Poisson form because (13) is time dependent, and thus the time derivative of a solution is not a solution.

Huygens' principle holds for this special initial condition because $\omega_{c t}[\phi]$ in $(16)$ is determined by the values of $\phi\left(x_{0}, y_{0}\right)$ on the surface but not in the interior of the 2-D light cone $\left(x-x_{0}\right)^{2}+\left(y-y_{0}\right)^{2}=c^{2} t^{2}$. Indeed, 14 
is the expanding-bubble wave created by a flash bulb. There is no ringing effect if $q=0$. (This is the reverse of what happens for the 1-D wave equation where Huygens' principle holds if $p=0$.)

Computational simulation of anomalous dispersion. The analytical arguments above can be verified by solving the equations numerically. We have used the differential equation solver Comsol Multiphysics to implement (1) for of three spatial dimensions [Fig. 1(a)], two spatial dimensions [Fig. 1(b)], and for (3) [Fig. 1) (c)].
A delta-function localized initial condition used in the derivations above in $(8)$ and $(10)$ ) cannot be modelled numerically. Instead, we use a gaussian distribution with a spatial width, details of which are given in the caption. Our simulations confirm the analytical results and show that solutions to the wave equation (1) in 3-D obey Huygens' principle, while in 2-D they exhibit anomalous dispersion. However, the modified 2-D wave equation (3) also obeys Huygens' principle with no anomalous dispersion and no trailing tail behind the pulses.
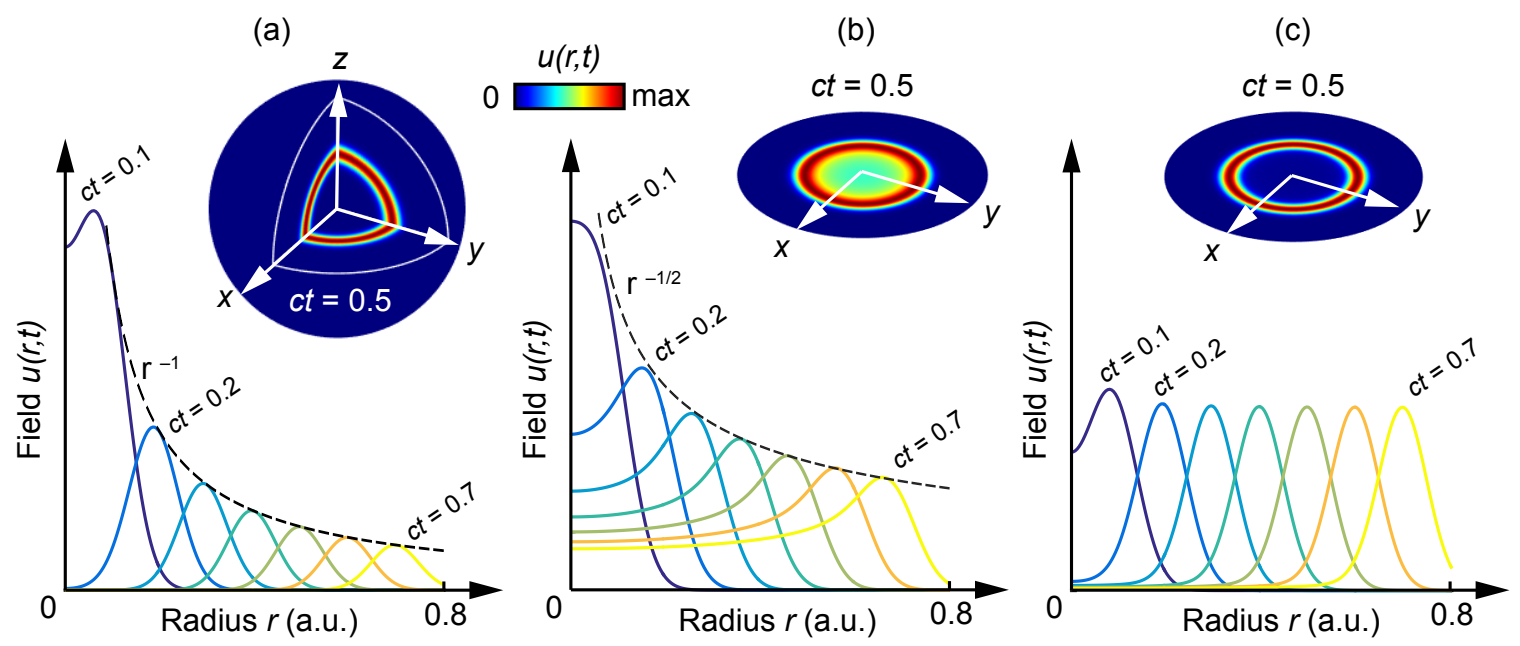

FIG. 1: [Color online] Numerical solution of the wave equation in three cases: (a) conventional 3-D wave equation $u_{t t}=$ $c^{2}\left(u_{x x}+u_{y y}+u z z\right)$; (b) conventional 2-D wave equation $u_{t t}=c^{2}\left(u_{x x}+u_{y y}\right)$ showing anomalous dispersion; (c) Modified two-dimensional wave equation $u_{t t}-(1 / t) u_{t}+\left(1 / t^{2}\right) u=c^{2}\left(u_{x x}+u_{y y}\right)$ with no anomalous dispersion. The insets show the solution at a fixed time. The initial conditions are: zero initial value $u(r, t=0)=0$ and a radially symmetric initial time derivative gaussian distribution $u_{t}(r, t=0)=\exp \left(-r^{2} /\left(2 \sigma_{r}^{2}\right)\right)$ with $\sigma_{r}=0.01$. The radial variable is $r=\sqrt{x^{2}+y^{2}+z^{2}}$ for $(\mathrm{a})$ and $r=\sqrt{x^{2}+y^{2}}$ for (b) and (c).

Wave equation with two space and two time dimensions. The change of variable $u(x, y, t)=t v(x, y, t)$ converts (3) to the wave equation

$$
v_{t t}+t^{-1} v_{t}=c^{2}\left(v_{x x}+v_{y y}\right) .
$$

The left side of this equation is the two-dimensional radial time derivative of the wave equation

$$
v_{\alpha \alpha}+v_{\beta \beta}=c^{2}\left(v_{x x}+v_{y y}\right)
$$

where $\mathbf{t}=(\alpha, \beta)$ is a two-dimensional vector time variable. This shows that anomalous dispersion does not occur for solutions to a linear homogeneous wave equation in a space of two time dimensions and two space dimensions as long as the initial disturbance has a vanishing derivative. Since the $3-\mathrm{D}$ wave equation takes the form in (17) inside hyperbolic metamaterials, these anisotropic media behave like a two-dimensional space with no anomalous dispersion. Experimental observation of the absence of anomalous dispersion in hyperbolic metamaterials is challanging because experiments to date have used narrowband sources. (Any source that is narrowband will have oscillations and the effect of this is to nearly cancel the $1 / t$ tail that is characteristic of anomalous dispersion in two-dimensional space.) However, recent developments in ultrashort broadband pulses allow for generation of even unipolar wave packets [10] which will be suitable for such experiments.) Theoretical considerations developed here explain for the first time how metamaterials can evade the anomalous dispersion intrinsic in 2-D space. 
* Electronic address: cmb@wustl.edu

$\dagger$ Electronic address: cisco.rodriguez fortuno@kcl.ac.uk

¥ Electronic address: sarben.sarkar@kcl.ac.uk

$\S$ Electronic address: a.zayats@kcl.ac.uk

[1] R. Courant and D. Hilbert, Methods of Mathematical Physics, Vol. 2 (Wiley, New York, 1962).

[2] P. R. Garabedian, Partial Differential Equations (Wiley, New York, 1964).

[3] I. I. Smolyaninov and E. E. Narimanov, Phys. Rev. Lett. 105, 067402 (2010).

[4] A. Poddubny, I. Iorsh, P. Belov, and Y. Kivshar, Nat. Photonics 7, 948 (2013).
[5] P. V. Kapitanova, P. Ginzburg, F. J. Rodríguez-Fortuño, D. S. Filonov, P. M. Voroshilov, P. A. Belov, A. N. Poddubny, Yu. S. Kivshar, G. A. Wurtz, and A. V. Zayats, Nat. Commun. 5, 3226 (2014).

[6] S. Ishii, A. V. Kildishev, E. Narimanov, V. M. Shalaev, and V. P. Drachev, Laser Photon. Rev. 7, 265 (2013).

[7] I. I. Smolyaninov, Y.-J. Hung, and E. E. Narimanov, Phys. Lett. A 376, 2575 (2012).

[8] I. I. Smolyaninov and Y.-J. Hung, Phys. Lett. A 377, 353 (2013)

[9] I. Bars and J. Terning, Extra dimensions in space and time (Sringer, New York, 2010).

[10] R. M. Arkhipov, A. V. Pakhomov, I. V. Babushkin, M. V. Arkhipov, Yu. A. Tolmachev, and N. N. Rosanov, J. Opt. Soc. Am. B 33, 2518 (2016). 